\title{
Expression and inhibitory role of TIMP-3 in hepatocellular carcinoma
}

\author{
BO SHEN $^{1}$, YINGJIE JIANG ${ }^{1}$, YUAN-RAN CHEN ${ }^{2}$, HUI-CONG ZHENG ${ }^{1}$, \\ WEI ZENG ${ }^{1}$, YU-YUAN LI ${ }^{1}$, AOXIAN YIN ${ }^{1}$ and YUQIANG NIE ${ }^{1}$ \\ ${ }^{1}$ Department of Gastroenterology and Hepatology, Guangzhou First People's Hospital, \\ Guangzhou Medical University; ${ }^{2}$ Department of Gastroenterology, Guangzhou Red Cross Hospital, \\ Ji'nan University, Guangzhou, Guangdong 510180, P.R. China
}

Received January 28, 2016; Accepted March 4, 2016

DOI: $10.3892 /$ or.2016.4818

\begin{abstract}
As a member of the tissue inhibitor of metalloproteinases (TIMP) family, it has been reported that TIMP-3 is involved in human cancer development. However, the function of TIMP-3 in hepatocellular carcinoma (HCC) development is unclear. We aimed to determine the biological role of TIMP-3 in HCC by evaluating the effects of its methylation status and expression on HCC cell function. TIMP-3 expression in HCC tissues was visibly analyzed by immunohistochemistry. Methylation of the TIMP-3 promoter was evaluated by methylation-specific PCR. Effects of TIMP-3 on HCC cell growth, apoptosis, migration, and invasion were examined by transfecting the TIMP-3-expressing plasmid, pCMV6. TIMP-3 was expressed in non-tumorous live tissue, but silenced or downregulated in $60 \%$ of $\mathrm{HCC}$ cases $(\mathrm{P}<0.05)$. Reduced protein expression of TIMP-3 was associated with reduced tumor differentiation $(\mathrm{P}=0.003)$ and increased metastatic activity $(\mathrm{P}=0.005)$ in $\mathrm{HCC}$ cell lines. Promoter methylation contributed to the TIMP-3 inactivation. Overexpression of TIMP-3 in HCC cell lines suppressed cell proliferation, induced apoptosis, and inhibited migration and invasion in vitro. TIMP-3 expression is suppressed by promoter methylation in HCC. This inhibitory protein acts as a functional tumor suppressor by inhibiting HCC cell proliferation, invasion, and migration and by inducing apoptosis and cell cycle arrest at the G2/M phase.
\end{abstract}

\section{Introduction}

Hepatocellular carcinoma (HCC) is one of the most malignant cancers, which accounts for almost $80 \%$ of all primary liver

Correspondence to: Dr Yuqiang Nie, Department of Gastroenterology and Hepatology, Guangzhou First People's Hospital, Guangzhou Medical University, 1 Panfu Road, Guangzhou, Guangdong 510180, P.R. China

E-mail: nieyq@medmail.com.cn

Key words: TIMP-3, hepatocellular carcinoma, matrix metalloproteases, methylation-specific PCR, apoptosis tumors (1). Despite effective surgical and drug therapy for HCC, such as surgical resection, partial melting and intervention, chemoradiotherapy, and other comprehensive treatment, the 5-year survival rate for HCC still remains very low. This high mortality rate is attributable mainly to tumor recurrence and metastases (2). Even with liver transplantation, the prognosis remains poor, owing to persistent tumor recurrence. Thus, there is a compelling need for a novel and specific therapeutic target.

Human tissue inhibitor of metalloproteinase-3 (TIMP-3), located on chromosome $22 \mathrm{q} 12$ (3) is an endogenous inhibitor of matrix metalloproteinases (MMPs), including metalloproteinases that have a disintegrin and metalloproteinase (ADAM) domain, and ADAMs with thrombospondin (TS)-like domains. These enzymes play important roles in degrading extracellular matrix (ECM) substrates (4). Unlike other TIMP variants (TIMP-1, 2, and 4), TIMP-3 has poor aqueous solubility and is a component of the ECM (5). Studies have demonstrated that TIMP-3 might function as a potential tumor suppressor gene through induction of tumor cell apoptosis, prevention of tumor ECM remodeling, and inhibition of tumor-derived angiogenic activity $(5,6)$

The expression of TIMP-3 is silenced in various types of malignant carcinoma (7). Regulation of TIMP-3 expression may be influenced by many upstream and downstream transcription factors. The type II nuclear receptor, peroxisome proliferator-activated receptor gamma, can upregulate TIMP-3 expression, which can be detected by cDNA microarray and chromatin immunoprecipitation coupled to PCR (8). Transcriptional reprogramming of tumors by overexpression of dormancy-associated microRNAs (DmiRs), particularly DmiR-580, 588, or 190, led to downregulation of TIMP-3 (9). Many other miRs can downregulate TIMP-3 expression, such as miR-21 (10), miR-103 (11), miR-181b (12), miR-181a (13), and miR-221/222 (14). These miRs all regulate TIMP-3 expression by targeting $\mathrm{mRNAs}$ at the 3'-untranslated regions (UTRs) for cleavage or translational repression. Thus, miR inhibition can significantly upregulate TIMP-3 protein expression in cancer cells (11).

Another mechanism by which TIMP-3 expression is regulated is the methylation of $\mathrm{CpG}$ islands in the TIMP-3 promoter, which results in a dramatic decrease in TIMP-3 mRNA levels $(15,16)$. Ten-eleven translocation methylcyto- 
sine dioxygenase 1 , encoded by the gene, TET1, has been shown to suppress tumor growth by downregulating TIMP-3 methylation (17). Masson et al showed that loss of TIMP-3 expression in clear cell renal cell carcinoma results from multiple mechanisms, including promoter hypermethylation. Methylation-associated inactivation of TIMP-3 has been reported in bladder cancer and in esophageal and gastric adenocarcinomas as well as renal cancer, and it is associated with poor tumor differentiation, high incidence of tumor invasiveness, and poor clinical outcome (18-20).

Invasion and metastasis of tumor cells are the key characteristics of malignant tumors. By binding to MMPs, in tumor cells, TIMP-3 inhibits their ECM-degrading activity, and thus TIMP-3 is crucially involved in the balance between synthesis and degradation of the ECM, a process which is closely related to tumor cell invasion and metastasis (21). Furthermore, the balance between MMPs and TIMPs is strongly linked to cellular proliferation, apoptosis, and cell cycle arrest as well as invasion and metastasis (22). The activities of both MMP-2, which can promote $\alpha v \beta 3$ integrinmediated adhesion and migration by cleaving fibronectin (23), and MMP-9, which can induce cleavage of the cell-cell/cellmatrix adhesion molecule, CD44 (24), plays an important role in increasing the migration and/or invasion potential of several kinds of cancer cells. Downregulation of the gene that codes for a proliferation-inducing ligand (APRIL), which is typically overexpressed in most tumor cells, leads to reduced expression of MMP-2 and MMP-9 and enhancement of TIMP-3 and TIMP-4 expression (4).

It has been reported that suppression of MMP-9 can inhibit tumor invasion and migration via activating transforming growth factor- $\beta$ (25), and loss of TIMP-3 expression can result from inactivation of transforming growth factor- $\beta$ receptor II (18). Recent studies have also shown that TIMP-3 suppresses tumor invasion via the TNF $\alpha / N F-\kappa B / I L-6$ pathway (16). We have previously found that the transcription factor, peroxisome proliferator activated receptor gamma, partially inhibits HCC cell invasiveness and metastasis via binding directly to the TIMP-3 promoter and upregulating TIMP-3 protein expression (8).

Although TIMP-3 has been shown to inhibit tumor growth, invasion, and metastasis (26), the effects of this physiological inhibitor in HCC have not been fully characterized. In this study, our aim was to assess the methylation status of the TIMP-3 promoter and expression of TIMP-3 in HCC and elucidate the biological function of TIMP-3 in HCC by exogenously inducing TIMP-3 expression in liver cancer cells.

\section{Materials and methods}

Patient tissue samples. Tissue samples embedded in paraffin blocks were obtained from the livers of $80 \mathrm{HCC}$ patients and 60 cancer-free patients (control subjects). None of these patients had received any other therapeutic intervention prior to surgery. Pertinent demographic and clinicopathological data, such as gender, age, tumor size, hepatitis B virus (HBV) infection, liver cirrhosis, tumor pathological grade, lymph node metastasis, and distant metastasis were collected for further analyses. The study protocol was approved by the Clinical Research Ethics Committee of Guangzhou Medical University.
Immunohistochemistry (IHC). IHC staining was performed on $4-\mu \mathrm{m}$-thick paraffin sections using the TIMP-3 primary antibody (1:100 dilution; Santa Cruz Biotechnology, Dallas, TX, USA). Sections were washed in Tris-buffered saline and stained in a two-step process involving incubation with a horseradish peroxidase-conjugated antibody kit (1:2000 dilution, Santa Cruz Biotechnology), followed by incubation with the chromagen, 3,3'-diaminobenzidine (Dako, Glostrup, Denmark). TIMP-3 immunostaining was defined according to the intensity and percentage of TIMP-3- positive tumor cells. The staining intensity was scored as follows: 0 (negative), 1 (weakly positive), 2 (moderately positive), and 3 (strongly positive). The percentage of TIMP-3 positive cells was classified into 4 categories: score of 1 for $0-10 \%, 2$ for $11-50 \%, 3$ for $51-80 \%$, and 4 for $81-100 \%$. The degree of TIMP-3 staining was quantified by using a two-level grading system (with the intensity score added to the percentage score to produce a final score), as follows: $<3$, negative expression; and 3-9, positive expression.

Human HCC cell lines and culture. The human HCC HepG2 cell line was a gift from AiMing Li, Department of Digestive System, Southern Medical University. The human HCC SMMC-7721 cell line was purchased from the Experimental Animal Center of Sun Yat-Sen University. Cells were cultured in Dulbecco's modified Eagle's medium (DMEM) with $10 \%$ fetal bovine serum in $5 \% \mathrm{CO}_{2}$ at $37^{\circ} \mathrm{C}$.

Bisulfite conversion and methylation-specific PCR (MSP). MSP was performed as previously described (16) and was used to examine the DNA methylation status of the TIMP-3 promoter. Genomic DNA was extracted from 8 different HCC cell lines with NucleoSpin Tissue mini spin columns (Macherey-Nagel GmbH \& Co. KG, Düren, Germany). Sodium bisulfite modification was performed with the EpiTect Bisulfie kit (Qiagen, Hilden, Germany). The primers used in MSP were designed via the MethPrimer web site (http://www.urogene. org/methprimer.html) and synthesized by Invitrogen Custom DNA Oligos (Life Technologies, Grand Island, NY, USA). The sequences of the forward and reverse primers were: MSP forward methylation primer, 5'-TCGAGGATTTAGCGGT AAGTATC-3'; MSP reverse methylation primer, 5'-GAA AACAAAAAATAACGAAACGAA-3'; MSP forward non-methylation primer, 5'-TTGAGGATTTAGTGGTAAG TATTGG-3'; MSP reverse non-methylation primer, 5'-CAA AAACAAAAAATAACAAAACAAA-3'. The MSP was carried out in the T100 Thermal Cycler (Bio-Rad, Hercules, CA, USA) under the following conditions: $30 \mathrm{sec}$ at $94^{\circ} \mathrm{C}$, $30 \mathrm{sec}$ at $54^{\circ} \mathrm{C}$, and $30 \mathrm{sec}$ at $72^{\circ} \mathrm{C}$ for 40 cycles, with a final 7 min extension at $72^{\circ} \mathrm{C}$. The PCR products were stained with ethidium bromide, followed by electrophoresis on $2 \%$ agarose gel and visualization under UV light. TIMP-3 mRNA was quantified by real-time PCR. Real-time amplification of $1 \mu \mathrm{g}$ of cDNA was performed with $12.5 \mu \mathrm{l}$ of Master SYBR Green Supermix in the CFX96 Touch Real-Time PCR Detection System (Bio-Rad). Amplification was performed, with the target gene mRNA levels being normalized to the expression of GAPDH. Results were analyzed by the $2^{-\Delta \mathrm{Ct}}$ method.

Demethylation treatment. Selected methylation-positive HCC cells were cultured with the DNA methyltransferase inhibitor, 
5-Aza-CdR, at a concentration of $5 \mu \mathrm{M}$ or $0 \mu \mathrm{M}$ (control). Pyrosequencing analysis was performed to examine the effects of 5-Aza-CdR on the methylation level of the TIMP-3 promoter $\mathrm{CpG}$ islands in $\mathrm{HCC}$ cells. Pyrosequencing was performed by the PyroMark Q96 ID System (Qiagen) as previously described (27).

Plasmids and transfection. HepG2 and SMMC-7721 cells were transfected with the pCMV6-TIMP-3 plasmid, which strongly expresses human TIMP-3 under a CMV promoter. The pCMV6-AC-GFP plasmid was used as the vector control. The open reading frames cloned in this vector are expressed in mammalian cells as a tagged protein (C-terminal tGFP), which can be visualized as green fluorescence under a fluorescence microscope. Since it has been shown that methylation of TIMP-3 in HepG2 is closely associated with TIMP-3 silencing in HCC and because the pCMV6-TIMP-3 plasmid has been successfully transfected into SMMC-7721 cells (28), we selected these cell lines for the transfection experiments. The cells were transfected using MegaTran 1.0 transfection reagent (OriGene, Rockville, MD, USA) according to the manufacturer's instructions. Cells were cultured for 2 weeks with the aminoglycoside, G418 (Thermo Fisher Scientific, Shanghai, China), to select the gene-transfected cells. Most cells are not transfected with the expression plasmid and thus are killed by G418. Those cells that remain growing in this selective medium have retained the expression plasmid, which stably integrates into the genome of the targeted cells. The group of cells transfected with the pCMV6-TIMP-3 plasmid were labeled as TIMP-3, and the group of cells not transfected with this plasmid were labeled as NC (control). The cultures were observed under fluorescence microscopy to confirm the presence of $\mathrm{tGFP}^{+}$transfected cells, and the transfection efficiency was determined by counting the number of $\mathrm{tGFP}^{+}$cells.

Western blot assay. Total protein was extracted and protein concentration was measured by the BCA protein assay (Bioworld, Minneapolis, MN, USA). Protein $(40 \mu \mathrm{g})$ from each sample was used for western blotting as previously described (29). Bands were observed using the Pierce Enhanced Chemiluminescence kit (Thermo Fisher Scientific).

Cell viability assay. Cell proliferation was determined by the 3-(4,5-dimethylthiazol-2-yl)-5-(3-carboxymethoxy-phenyl)2-(4-sulfophenyl)-2H-tetrazolium (MTS) assay (Promega, Madison, WI, USA). Briefly, cells (3000/well) were placed in 96-well plates. After $24 \mathrm{~h}, 20 \mu \mathrm{l}$ of MTS reaction solution was added to cultured cells in $100 \mu \mathrm{l}$ medium and incubated at $37^{\circ} \mathrm{C}$ for $1 \mathrm{~h}$. The optical density was measured at $490 \mathrm{~nm}$ in the Sunrise microplate absorbance reader (Tecan Group Ltd., Männedorf, Switzerland).

Cell apoptosis assay. Cell apoptosis was assessed using the Annexin V-FITC Apoptosis Detection Kit I (BD Pharmingen, San Diego, CA, USA) according to the manufacturer's instructions. Cells were washed twice with cold phosphate-buffered saline (PBS) and resuspended in $1 \mathrm{X}$ binding buffer. Next, $5 \mu \mathrm{l}$ of Annexin V-FITC and $5 \mu \mathrm{l}$ of propidium iodide (PI) staining solution were added, followed by incubation for $15 \mathrm{~min}$ in the
Table I. Comparison of TIMP-3 expression between HCC and control subjects.

\begin{tabular}{lcccc}
\hline Subjects & TIMP-3-positive & TIMP-3-negative & $\chi^{2}$ & P-value \\
\hline All cases & $69(49.29)$ & $71(50.71)$ & & \\
HCC & $32(40.00)$ & $48(60.00)$ & 6.439 & 0.011 \\
Control & $37(61.67)$ & $23(38.33)$ & & \\
\hline
\end{tabular}

${ }^{a}$ Values represent n (\%).

dark at room temperature $\left(25^{\circ} \mathrm{C}\right)$. Finally, cells were suspended in $400 \mu 1 \mathrm{XX}$ binding buffer and analyzed within $1 \mathrm{~h}$ on the FACSCalibur Flow Cytometer (BD Biosciences, San Jose, CA, USA). Experiments were conducted in triplicate.

Cell cycle assay. Cells were trypsinized, washed twice with cold PBS, and fixed for $1 \mathrm{~h}$ in ice-cold $70 \%$ ethanol. The cells were resuspended in cold PBS, and $20 \mu 1$ of RNase A solution were then added, followed by incubation for $30 \mathrm{~min}$ at $37^{\circ} \mathrm{C}$. Finally, cells were labeled with PI and analyzed on the FACSCalibur Flow Cytometer. Experiments were conducted in triplicate.

Invasion assay. The invasion assay was performed using transwell chambers (BD Biosciences) with polycarbonate membrane inserts $(8-\mu \mathrm{m}$ pore size). Diluted Matrigel (BD Biosciences), which acts as a chemoattractant, was added to the upper chamber surface. After $1 \mathrm{~h}$ of incubation at $37^{\circ} \mathrm{C}$, the cells $\left(2 \times 10^{4}\right.$ per well) were collected in serum-free medium and added to the upper chambers. The lower chamber contained $600 \mu 1$ DMEM containing 10\% fetal bovine serum. After $24 \mathrm{~h}$, cells that invaded through the Matrigel membrane were fixed in $10 \%$ formalin, stained with crystal violet, and counted under a microscope (4 high-power fields at x100 magnification). Experiments were conducted in triplicate.

Migration assay. In the migration assay, transwell chambers were also used $\left(2 \times 10^{4}\right.$ cells per well), but no Matrigel was used. Instead, the cells were collected and diluted in serumfree medium, and placed in the upper chambers. The lower chamber contained $600 \mu \mathrm{l}$ DMEM containing $10 \%$ fetal bovine serum. After $24 \mathrm{~h}$, cells that had moved to the bottom surface of the filter membrane were fixed in $10 \%$ formalin, stained with crystal violet, and counted under a microscope (4 high-power fields at x100 magnification). Experiments were conducted in triplicate.

Statistical analyses. Data are presented as the mean \pm standard deviation (SD). Differences between two groups were analyzed by Student's t-test. The correlation between TIMP-3 positive-expression and liver cancer was analyzed by the MannWhitney test. Chi-square tests were performed to evaluate the relationship between TIMP-3 expression and clinicopathological parameters. Statistical analyses were performed in the SPSS package for Windows (version 16.0; SPSS Inc., Chicago, IL, USA). Differences with a P-value $<0.05$ were considered statistically significant. 

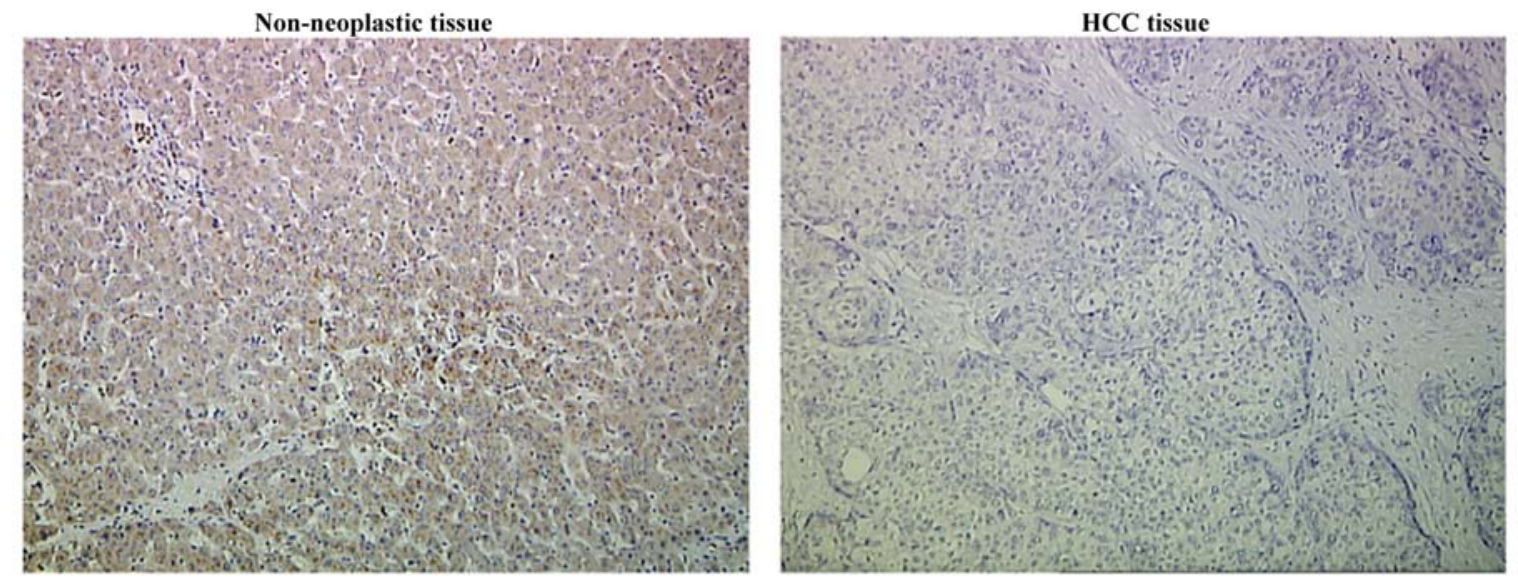

Figure 1. Representative photomicrographs of immunoreactive TIMP-3 in nonneoplastic tissue and HCC tissue. Immunostaining was decreased in the HCC tissue. Magnification, x100.

\section{Results}

TIMP-3 expression in HCC tissues. As shown in Table I and Fig. 1, TIMP-3 was expressed in only 32 of 80 (40\%) HCC tissue samples, whereas 37 of 60 tumor-free samples $(61.67 \%)$ exhibited TIMP-3 expression $(\mathrm{P}=0.011)$. Tissue from $76.67 \%$ of younger patients ( $<50$ years) with $\mathrm{HCC}$ showed negative TIMP-3 expression, while only $50 \%$ of tissues from older patients exhibited negative TIMP-3 expression $(\mathrm{P}=0.018)$. Reduced expression of TIMP-3 was correlated with higher frequencies of poorly differentiated carcinomas and metastasis. No significant correlations were observed between TIMP-3 expression and the remaining clinicopathological parameters (Table II).

TIMP-3 gene expression and methylation status in HCC cells. Results of MSP analysis were positive for TIMP-3 promoter methylation in 3 of 8 HCC cell lines: $\mathrm{C} 3 \mathrm{~A}, \mathrm{HepG} 2$, and Hep-3B (Fig. 2A). Thus, we selected these cell lines and treated them with 5-Aza-CdR. As shown in Fig. 2B, TIMP-3 mRNA in all three cell lines was upregulated by demethylation treatment $(\mathrm{P}<0.05)$. Results of the pyrosequencing analyses (Fig. 2C) revealed decreased methylation level in all three cell lines after 5-Aza-CdR treatment $(\mathrm{P}<0.05)$.

Expression of TIMP-3 plasmid in transfected HCC cells. An illustration of the GFP expression vector is shown in Fig. 3A, and the extent of GFP labeling in SMMC-7721 and HepG2 cells is shown in Fig. 3B. Nearly all the G418-selected cells exhibited green fluorescence. The western blots revealed robust TIMP-3 protein expression in the TIMP-3 group, whereas no protein expression was observed in the $\mathrm{NC}$ group (Fig. 3C).

Effects of TIMP-3 overexpression on HCC cell viability, apoptosis, and cell cycle arrest. The MTS assay revealed suppressed growth of SMMC-7721 and HepG2 cells, stably transfected with TIMP-3, in a significant, time-dependent manner (Fig. 4). As shown in Fig. 5A-C, Flow cytometric analysis of Annexin V-FITC/PI revealed an increased mean number of apoptotic cells in the TIMP-3 group compared to the NC group (SMMC-7721: $30.7 \pm 1.6 \%$ vs. $8.4 \pm 1.1 \%, \mathrm{P}<0.01$; HepG2: $12.6 \pm 1.1 \%$ vs. $7.6 \pm 1.3 \%, \mathrm{P}<0.05)$. The flow cytometry
Table II. Correlation of TIMP-3 expression with clinicopathological characteristics in HCC patients.

\begin{tabular}{|c|c|c|c|}
\hline Characteristics & $\begin{array}{c}\text { TIMP-3-positive } \\
(\%)\end{array}$ & $\begin{array}{c}\text { TIMP-3-negative } \\
(\%)\end{array}$ & P-value \\
\hline Gender & & & 0.598 \\
\hline Male & 25 (41.67) & $35(58.33)$ & \\
\hline Female & $7(35.00)$ & $13(65.00)$ & \\
\hline Age (years) & & & 0.018 \\
\hline$<50$ & $7(23.33)$ & 23 (76.67) & \\
\hline$\geq 50$ & $25(50.00)$ & $25(50.00)$ & \\
\hline Tumor size & & & 0.144 \\
\hline$<5 \mathrm{~cm}$ & $20(47.62)$ & $22(52.38)$ & \\
\hline$\geq 5 \mathrm{~cm}$ & $12(31.58)$ & $26(68.42)$ & \\
\hline Differentiation & & & 0.003 \\
\hline Mod-well & $23(56.10)$ & $18(43.90)$ & \\
\hline Low & $9(23.08)$ & $30(76.92)$ & \\
\hline Metastasis & & & 0.005 \\
\hline Yes & $11(25.59)$ & $32(74.41)$ & \\
\hline No & $21(56.76)$ & $16(43.24)$ & \\
\hline HBV infection & & & 0.205 \\
\hline Yes & $19(35.19)$ & $35(64.81)$ & \\
\hline No & $13(50.00)$ & $13(50.00)$ & \\
\hline Fatty liver & & & 0.059 \\
\hline Yes & $4(80.00)$ & $1(20.00)$ & \\
\hline No & $28(37.33)$ & 47 (62.67) & \\
\hline Cirrhosis & & & 0.923 \\
\hline Yes & $21(39.62)$ & $32(60.38)$ & \\
\hline No & $11(40.74)$ & $16(59.26)$ & \\
\hline
\end{tabular}

results depicted in Fig. 6A, band c revealed an induction of cell cycle arrest in TIMP- 3 transfected cells, as evidenced by a decreased mean number of $\mathrm{G}_{0} / \mathrm{G}_{1}$ cells compared to that of the NC group (SMMC-7721: $36.08 \pm 3.53 \%$ vs. $57.72 \pm 1.53 \%$, $\mathrm{P}<0.05$; HepG2: $49.82 \pm 1.46 \%$ vs. $59.47 \pm 1.27 \%, \mathrm{P}<0.05)$ and 
A

\begin{tabular}{|c|c|c|c|c|c|c|c|c|c|c|c|c|c|c|c|}
\hline $\mathrm{C} 3 \mathrm{~A}$ & & $\mathrm{RF} / \mathrm{S}$ & & h-7 & & & & 402 & & G2 & SMMO & 721 & QG & 7701 & blank \\
\hline M & M & $\mathrm{U}$ & M & $\mathrm{U}$ & M & $\mathrm{U}$ & M & $\mathbf{U}$ & M & U & M & U & M & $\mathbf{U}$ & M \\
\hline
\end{tabular}

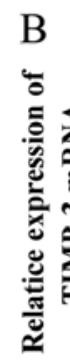

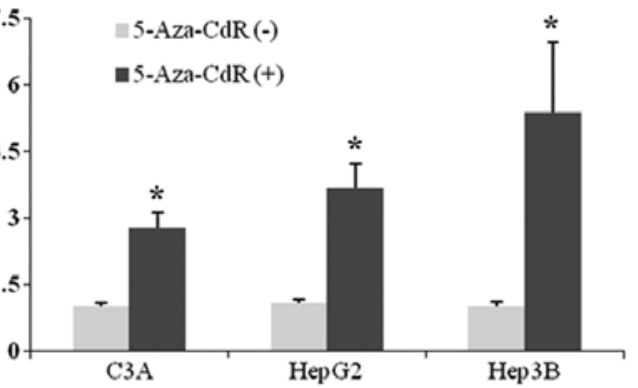

TIMP-3 expression

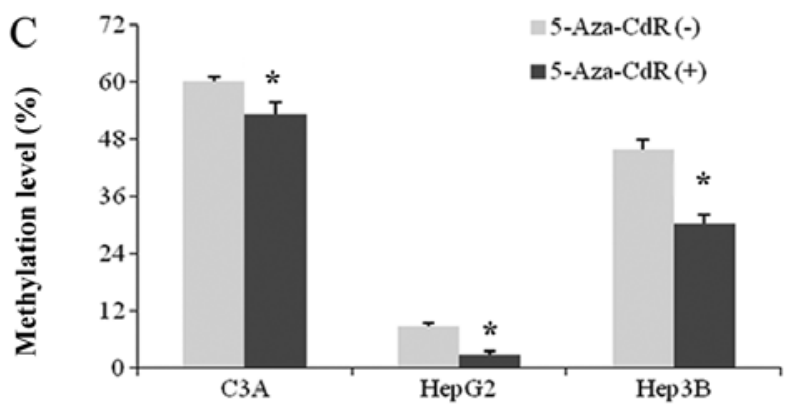

TIMP-3 methylation

Figure 2. TIMP-3 gene expression and promoter methylation in HCC cell lines. (A) Representative agarose gel showing TIMP-3 promoter DNA bands in 8 HCC cell lines. M, PCR specific for methylated DNA; U, PCR specific for unmethylated DNA. (B) Relative TIMP-3 gene expression in response to 5-AzaCdRin 3 HCC cell lines. " $\mathrm{P}<0.05$ vs. untreated group. (C) Methylation level in response to 5-Aza-CdR in the same HCC cell lines. " $\mathrm{P}<0.05$ vs. untreated group.

A

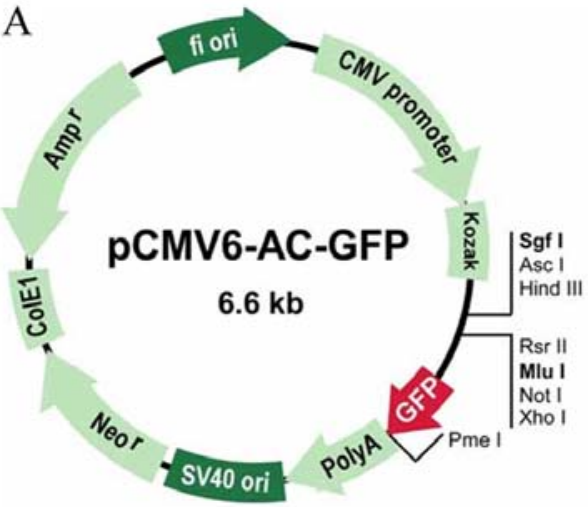

$\mathrm{C}$

TIMP-3

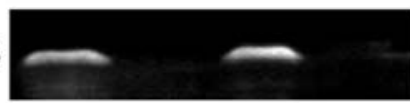

$\beta$-actin
B
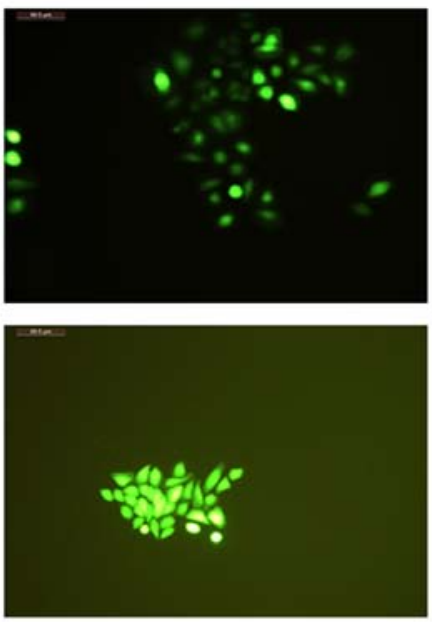
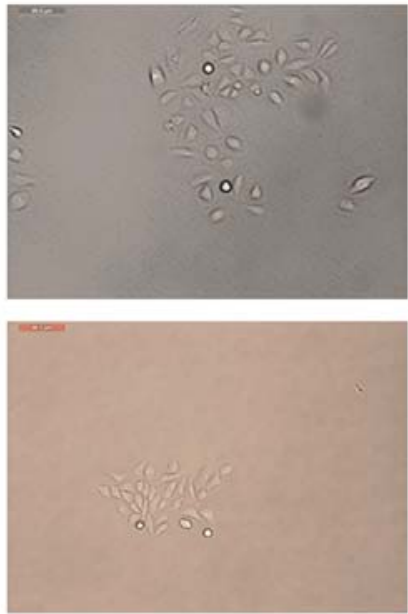

Figure 3. Expression of transfected TIMP-3 gene in HCC cells. (A) Vector map of pCMV6-AC-GFP plasmid. (B) Representative fluorescence (left) and nonfluorescence (right) photomicrographs of tGFP+ positive SMMC-7721 and HepG2 cells transfected with TIMP-3 plasmid. Cells appear as fluorescent green dots in fluorescence photomicrograph. Magnification, x100. (C) Representative western blots showing expression of TIMP-3 protein in the following lanes: 1, SMMC-7721, TIMP-3 group; 2, SMMC-7721, NC group; 3, HepG2, TIMP-3 group; 4, HepG2, NC group. $\beta$-actin was used as the loading control.
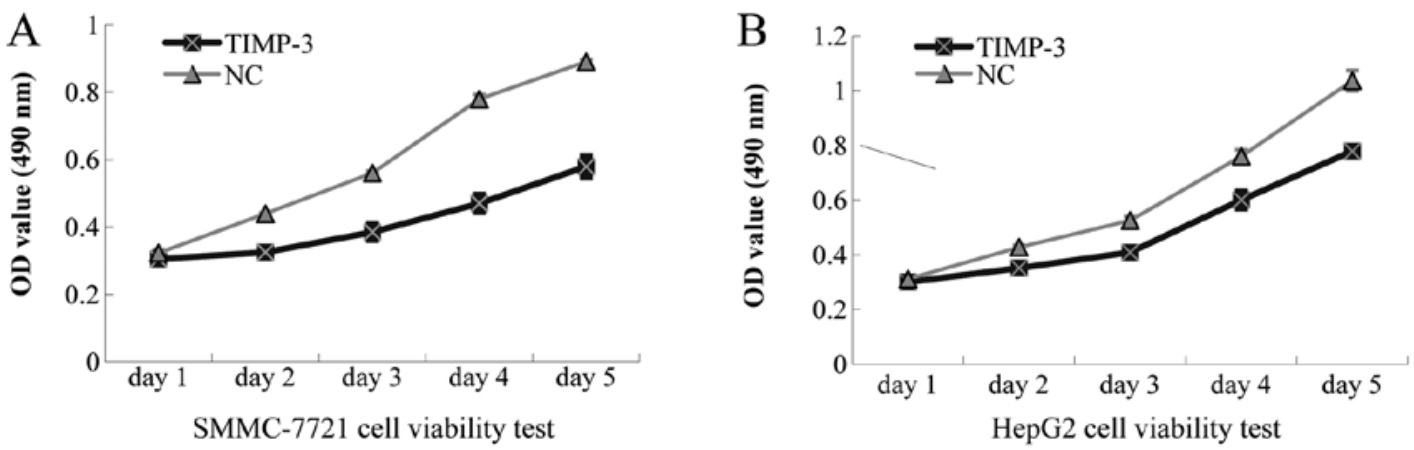

Figure 4. Time course of cell viability measured as MTS reduction in TIMP-3-transfected SMMC-7721 (A) and HepG2 (B) cells. "P<0.05 vs. NC group at day 3 . ${ }^{* *} \mathrm{P}<0.01$ vs. NC group at day 5 . 

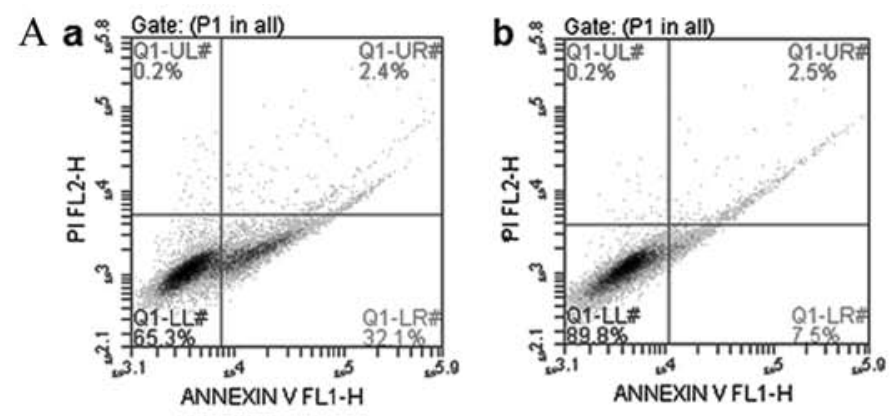

B

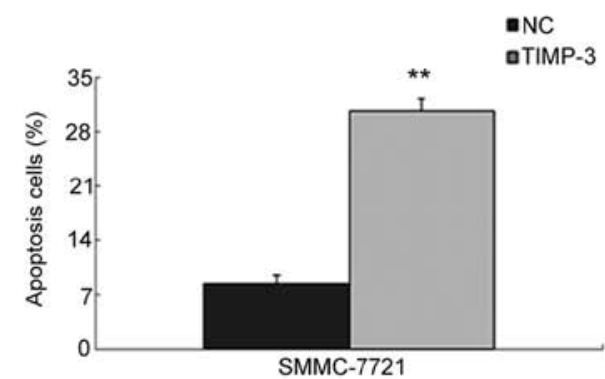

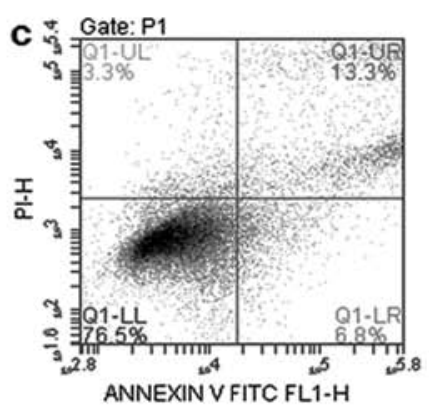

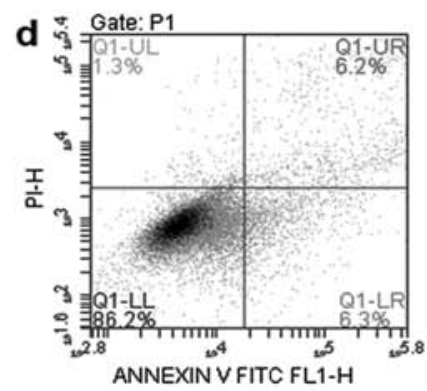

C

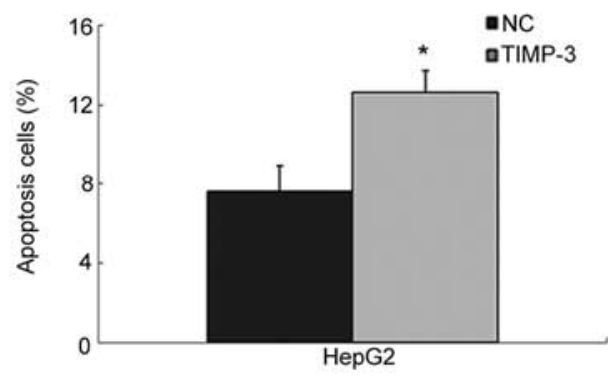

Figure 5. TIMP-3 induces cell apoptosis. (A) Flow cytometry analysis of apoptotic HCC cells transfected with TIMP-3. Cells were stained with Annexin V-FITC/PI. The dot plots represent the following cells: (a) SMMC-7721, TIMP-3 group; (b) SMMC-7721, NC group; (c) HepG2, TIMP-3 group; (d) HepG2, NC group. Column graphs of the flow cytometry data from SMMC-7721. (B) and HepG2 (C) cells. Each column represents the mean \pm SD of 3 experiments. ${ }^{*} \mathrm{P}<0.05$ vs. $\mathrm{NC}$ group. ${ }^{* *} \mathrm{P}<0.01$ vs. $\mathrm{NC}$ group.

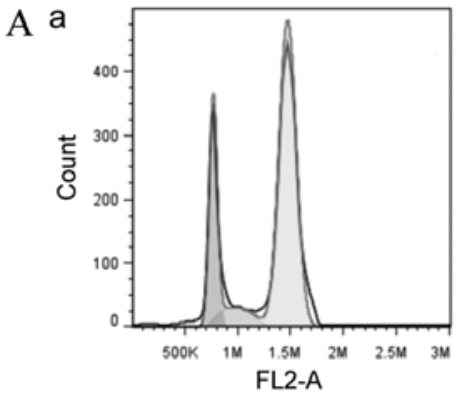

B

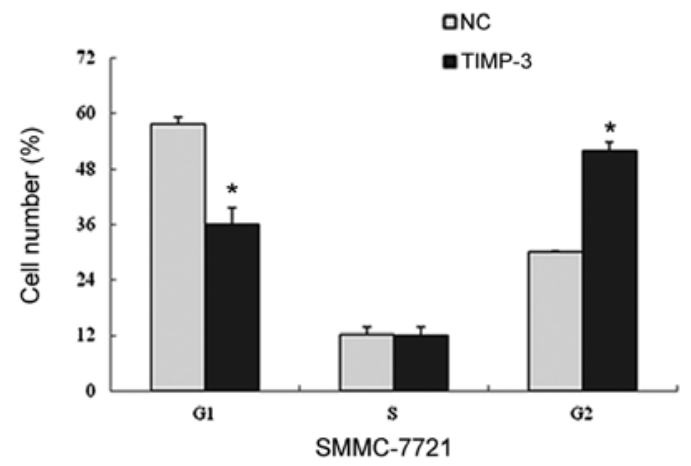

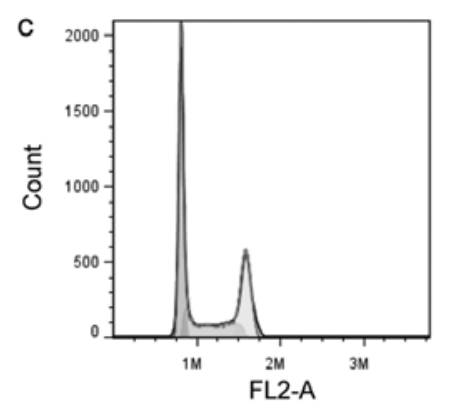

$\mathrm{C}$
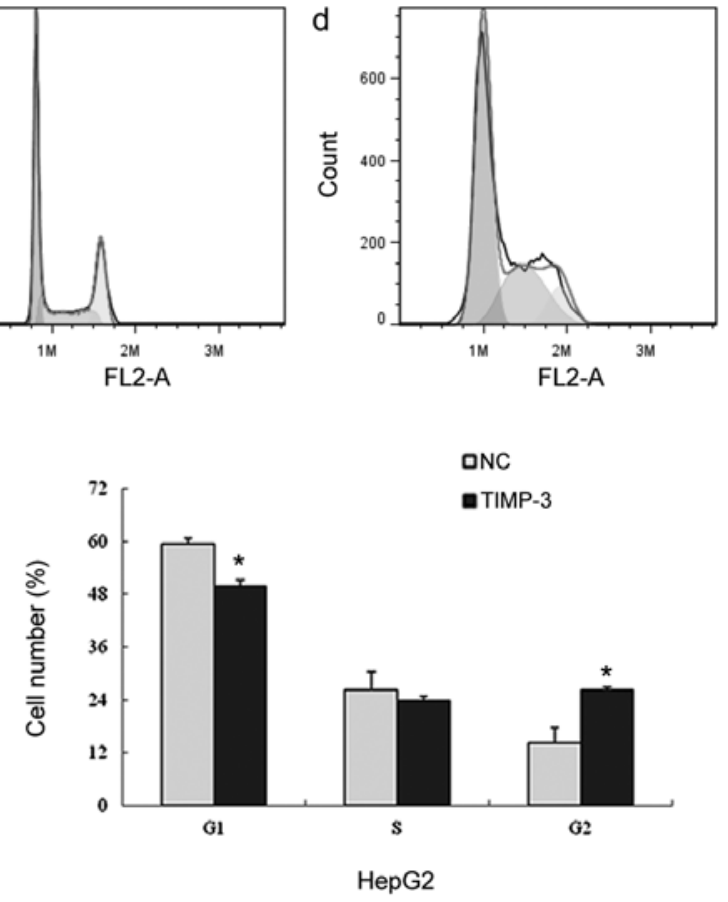

QNC

HepG2

Figure 6. TIMP-3 induces cell cycle arrest. (A) Representative flow cytometry histograms showing cell cycle phases in the following cells: (a) SMMC-7721, TIMP-3 group; (b) SMMC-7721, NC group; (c) HepG2, TIMP-3 group; (d) HepG2, NC group. Column graphs of the flow cytometry data from SMMC-7721 (B) and HepG2 (C) cells. For each cell cycle phase, each column represents the mean \pm SD of 3 experiments. ${ }^{*} \mathrm{P}<0.05$ vs. NC group.

an increased mean number of $\mathrm{G}_{2} / \mathrm{M}$ cells compared to that of the NC group (SMMC-7721: $51.91 \pm 1.71 \%$ vs. $30.11 \pm 0.20 \%$, $\mathrm{P}<0.05$; HepG2: $26.27 \pm 0.54 \%$ vs. $14.15 \pm 3.61 \%, \mathrm{P}<0.05)$.

Effect of TIMP-3 on SMMC-7721 and HepG2 invasion and migration. The number of cells penetrating through the Matrigel membrane reflects the cell invasion ability. As shown in Fig. 7A, the mean number of TIMP-3-transfected invading cells was lower compared to that of the NC group (SMMC7721: $60 \pm 11$ vs. $121 \pm 17, \mathrm{P}<0.05$; HepG2: $98 \pm 6$ vs. $192 \pm 22$, $\mathrm{P}<0.05$ ). Results of the migration assay (Fig. 7B) revealed a lower mean number of TIMP-3 transfected migrating cells compared to that of the NC group (SMMC-7721: 53 \pm 8 vs. $102 \pm 10, \mathrm{P}<0.01$; HepG2: $109 \pm 9$ vs. $232 \pm 13, \mathrm{P}<0.01$ ). 
A
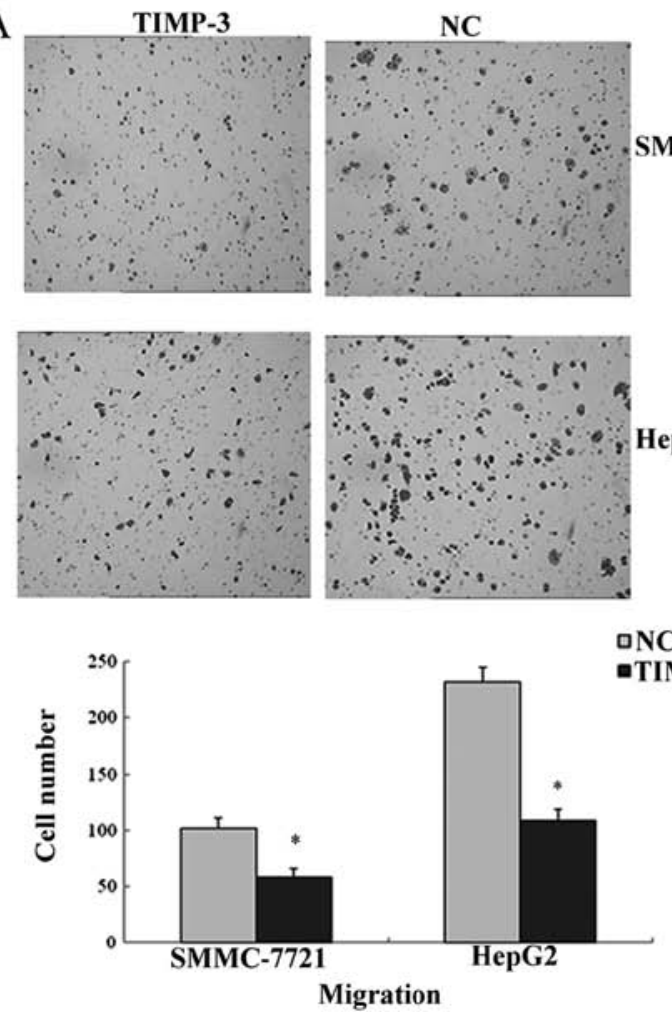

B
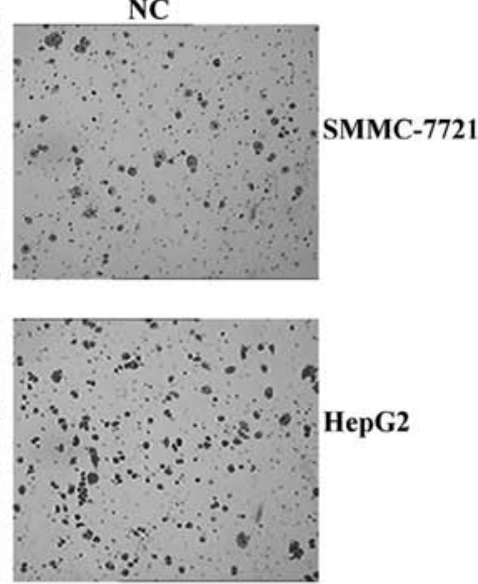

NC

TIMP-3
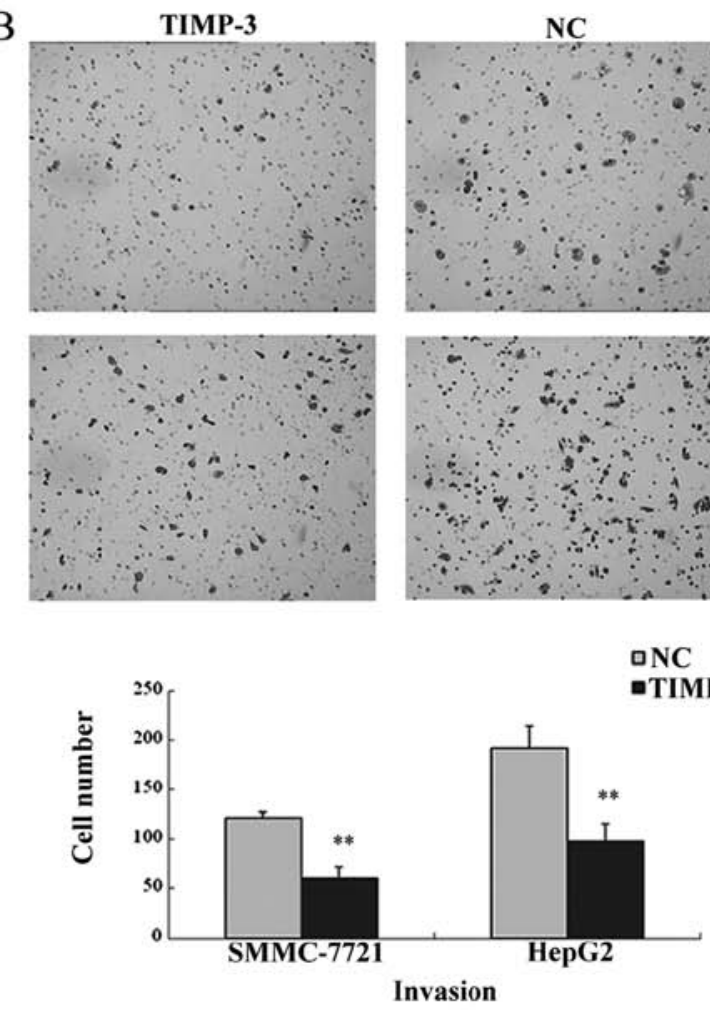

Figure 7. TIMP-3 effect on SMMC-7721 and HepG2 cells invasion and migration. (A) Representative photomicrographs of the following invading cells from the Matrigel-coated transwell assay (x100), and the column graphs of invasion. (B) Representative micrographs of the following migrating cells from the transwell assay (x100), and the column graphs of migration. Each column represents the mean $\pm \mathrm{SD}$ of 3 experiments. ${ }^{*} \mathrm{P}<0.05$ vs. NC group. ${ }^{* *} \mathrm{P}<0.01$ vs. NC group.

\section{Discussion}

In this study, we characterized the methylation status of the TIMP-3 promoter in HCC cells and have examined the effects of TIMP-3 promoter methylation and TIMP-3 expression on several parameters of HCC cell function. The results from our study allow us to elucidate the biological role of TIMP-3 in HCC and the molecular basis for the TIMP-3 effects on HCC cells. We found decreased TIMP-3 expression in a significant number of tissue samples from patients with HCC. Methylation status of the TIMP-3 promoter was positive in three HCC cell lines (C3A, HepG2, and Hep-3B), and the expression of TIMP-3 mRNA significantly increased after demethylation with 5-Aza-CdR. These results indicate that TIMP-3 expression in HCC is downregulated, and the mechanism responsible for this downregulation, at least in part, is methylation of the TIMP-3 promoter. These findings are similar to those of other studies that revealed methylation of TIMP-3 in brain, lung, kidney, colorectal, and other tumors $(7,30,31)$.

To determine whether TIMP-3 has antitumor activity in HCC, we upregulated TIMP-3 expression in HCC cells via gene transfection. After incubation with selective media, nearly all the remaining cells expressed tGFP, as evidenced by the observed green fluorescence. This indicates that the TIMP-3 plasmid was transfected with relatively high efficiency. These transfected HCC cells exhibited robust expression of TIMP-3 protein, which was associated with decreased cell proliferation. Upregulation of TIMP-3 expression results in significantly increased expression of cyclin-dependent kinase inhibitor, p27 (30). It has been shown that p27, by binding to and inhibiting cyclin-cyclin-dependent kinase complexes directly involved in cell cycle control $(31,32)$, is a key inhibitor of cell proliferation. The loss of p27 expression promotes hepatocarcinogenesis through STAT3 signaling activation (33).

Along with reduced cell proliferation, we also observed a significant increase in apoptosis in TIMP-3 transfected HCC cells. There are two major apoptotic pathways that cells can follow: the extrinsic Fas death receptor-mediated pathway and the intrinsic, mitochondrial-associated caspase-dependent pathway. Binding of the Fas receptor to its ligand (Fas-L) can trigger a signaling cascade via the intrinsic caspasedependent pathway that results in apoptosis $(34,35)$, and it has been shown that TIMP-3 can induce this intrinsic apoptotic pathway that is initiated via the extrinsic Fas-associated mechanism (17). Another study demonstrated that increased expression of TIMP-3, via silencing the APRIL gene, leads to decreased expression of the antiapoptotic proteins, Bcl-2 and $\mathrm{Bcl}-\mathrm{xL}$, and increased expression of the proapoptotic protein, Bax (36). Additional evidence suggests that TIMP-3-induced upregulation of p27 can result in increased expression of apoptosis-related proteins (33).

We used flow cytometry analysis to evaluate the effects of TIMP-3 on cell cycling. The decreased number of $\mathrm{G}_{0} / \mathrm{G}_{1}$ and increased number $\mathrm{G}_{2} / \mathrm{M}$ TIMP-3-transfected HCC cells suggest that TIMP-3 can induce $\mathrm{G}_{2} / \mathrm{M}$ phase arrest, with a concomitant reduction in cellular proliferation. $\mathrm{G}_{2} / \mathrm{M}$ arrest by TIMP-3 is linked to 553 , which controls the entry of cells into the mitosis phase at the $G_{2}$ checkpoint (37). Cells prepare for 
mitosis by increasing the level of cyclin B1 and activating $\mathrm{Cdc} 2$, the cyclin-dependent kinase required to enter mitosis (36). The mechanism by which p53 blocks entry into the mitosis phase partly involves inhibition of $\mathrm{Cdc} 2$ by several transcription factors: p21, 14-3-3, and Gadd45. p21 can bind to and inhibit the cyclin B1/Cdc2 complex, thereby inactivating $\mathrm{Cdc} 2$ (38). When the cyclin B1-Cdc2 complexes are inactivated, the cells are arrested in the $\mathrm{G}_{2} / \mathrm{M}$ phase (39).

Invasion and migration ability are typical features of HCC and other malignant cell types. Compared to non-transfected cells, we observed significantly lower numbers of transfected HCC cells that were able to penetrate through the Matrigel and cross the membrane to the lower chamber. These results indicate that increased expression of TIMP-3 suppresses the HCC cell ability to metastasize and invade surrounding tissues. As observed for other kinds of malignant tumors, our study provides evidence that TIMP-3 has antitumor activity against HCC and that TIMP-3 may exert this antitumor activity via multiple mechanisms.

Having observed the TIMP-3 protein expression and suppressive function clinically and in vitro, we plan to explore the molecular mechanism underlying the inhibitory effect of TIMP-3 on HCC in our next project. TIMP-3 is considered a tumor suppressor gene that may be capable of upregulating other antitumorigenic genes. Thus, the precise downstream pathways by which it mediates such effects are worthy of future studies.

In conclusion, $T I M P-3$ can act as a tumor-suppressor gene in HCC. TIMP-3 is expressed at low levels in HCC, but its expression can be activated by treatment with demethylation agents, such as 5-Aza-CdR. TIMP-3 exerts its anticancer effects on HCC via suppressing cell proliferation, inducing apoptosis and $\mathrm{G}_{2} / \mathrm{M}$ phase arrest, and inhibiting invasion and migration.

\section{Acknowledgements}

The study was supported by the National Natural Science Foundation of China (no. 81270037), the Research Fund for the Doctoral Program of Higher Education of China (no. 20114423110005) and Medical Scientific Research Foundation of Guangdong Province (no. A2014550).

\section{References}

1. French SW, Lee J, Zhong J, Morgan TR, Buslon V, Lungo W and French BA: Alcoholic liver disease - Hepatocellular carcinoma transformation. J Gastrointest Oncol 3: 174-181, 2012.

2. Mann CD, Neal CP, Garcea G, Manson MM, Dennison AR and Berry DP: Prognostic molecular markers in hepatocellular carcinoma: A systematic review. Eur J Cancer 43: 979-992, 2007.

3. Apte SS, Mattei MG and Olsen BR: Cloning of the cDNA encoding human tissue inhibitor of metalloproteinases-3 (TIMP-3) and mapping of the TIMP3 gene to chromosome 22 Genomics 19: 86-90, 1994.

4. Yu WH, Yu S, Meng Q, Brew K and Woessner JF Jr: TIMP-3 binds to sulfated glycosaminoglycans of the extracellular matrix. J Biol Chem 275: 31226-31232, 2000.

5. Anand-Apte B, Bao L, Smith R, Iwata K, Olsen BR, Zetter B and Apte SS: A review of tissue inhibitor of metalloproteinases-3 (TIMP-3) and experimental analysis of its effect on primary tumor growth. Biochem Cell Biol 74: 853-862, 1996.

6. Kallio JP, Hopkins-Donaldson S, Baker AH and Kähäri VM: TIMP-3 promotes apoptosis in nonadherent small cell lung carcinoma cells lacking functional death receptor pathway. Int $\mathbf{J}$ Cancer 128: 991-996, 2011.
7. Bachman KE, Herman JG, Corn PG, Merlo A, Costello JF, Cavenee WK, Baylin SB and Graff JR: Methylation-associated silencing of the tissue inhibitor of metalloproteinase-3 gene suggest a suppressor role in kidney, brain, and other human cancers. Cancer Res 59: 798-802, 1999.

8. Shen B, Chu ES, Zhao G, Man K, Wu CW, Cheng JT, Li G, Nie Y, Lo CM, Teoh N, et al: PPARgamma inhibits hepatocellular carcinoma metastases in vitro and in mice. $\mathrm{Br} \mathrm{J}$ Cancer 106: 1486-1494, 2012

9. Almog N, Ma L, Schwager C, Brinkmann BG, Beheshti A, Vajkoczy P, Folkman J, Hlatky L and Abdollahi A: Consensus microRNAs governing the switch of dormant tumors to the fastgrowing angiogenic phenotype. PLoS One 7: e44001, 2012.

10. Nagao Y, Hisaoka M, Matsuyama A, Kanemitsu S, Hamada T, Fukuyama T, Nakano R, Uchiyama A, Kawamoto M, Yamaguchi K, et al: Association of microRNA-21 expression with its targets, PDCD4 and TIMP3, in pancreatic ductal adenocarcinoma. Mod Pathol 25: 112-121, 2012.

11. Yu D, Zhou H, Xun Q, Xu X, Ling J and Hu Y: microRNA-103 regulates the growth and invasion of endometrial cancer cells through the downregulation of tissue inhibitor of metalloproteinase 3. Oncol Lett 3: 1221-1226, 2012.

12. Guo JX, Tao QS, Lou PR, Chen XC, Chen J and Yuan GB: miR-181b as a potential molecular target for anticancer therapy of gastric neoplasms. Asian Pac J Cancer Prev 13: 2263-2267, 2012.

13. Panda H, Chuang TD, Luo $X$ and Chegini N: Endometrial miR-181a and miR-98 expression is altered during transition from normal into cancerous state and target PGR, PGRMC1, CYP19A1, DDX3X, and TIMP3. J Clin Endocrinol Metab 97: E1316-E1326, 2012.

14. Zhang C, Zhang J, Hao J, Shi Z, Wang Y, Han L, Yu S, You Y, Jiang $\mathrm{T}$, Wang J, et al: High level of miR-221/222 confers increased cell invasion and poor prognosis in glioma. J Transl Med 10: 119, 2012.

15. Lü GL, Wen JM, Xu JM, Zhang M, Xu RB and Tian BL: Relationship between TIMP-3 expression and promoter methylation of TIMP-3 gene in hepatocellular carcinoma. Zhonghua Bing Li Xue Za Zhi 32: 230-233, 2003 (In Chinese).

16. Lee S, Lee HJ, Kim JH, Lee HS, Jang JJ and Kang GH: Aberrant $\mathrm{CpG}$ island hypermethylation along multistep hepatocarcinogenesis. Am J Pathol 163: 1371-1378, 2003.

17. Bond M, Murphy G, Bennett MR, Newby AC and Baker AH: Tissue inhibitor of metalloproteinase-3 induces a Fas-associated death domain-dependent type II apoptotic pathway. J Biol Chem 277: 13787-13795, 2002.

18. Masson D, Rioux-Leclercq N, Fergelot P, Jouan F, Mottier S, Théoleyre S, Bach-Ngohou K, Patard JJ and Denis MG: Loss of expression of TIMP3 in clear cell renal cell carcinoma. Eur J Cancer 46: 1430-1437, 2010.

19. Hoque MO, Begum S, Brait M, Jeronimo C, Zahurak M, Ostrow KL, Rosenbaum E, Trock B, Westra WH, Schoenberg M, et al: Tissue inhibitor of metalloproteinases-3 promoter methylation is an independent prognostic factor for bladder cancer. J Urol 179: 743-747, 2008.

20. Gu P, Xing X, Tänzer M, Röcken C, Weichert W, Ivanauskas A, Pross M, Peitz U, Malfertheiner P, Schmid RM, et al: Frequent loss of TIMP-3 expression in progression of esophageal and gastric adenocarcinomas. Neoplasia 10: 563-572, 2008.

21. Stetler-Stevenson WG: The role of matrix metalloproteinases in tumor invasion, metastasis, and angiogenesis. Surg Oncol Clin N Am 10: 383-392, x, 2001.

22. Amălinei C, Căruntu ID, Giuscă SE and Bălan RA: Matrix metalloproteinases involvement in pathologic conditions. Rom J Morphol Embryol 51: 215-228, 2010.

23. Jiao Y, Feng X, Zhan Y, Wang R, Zheng S, Liu W and Zeng X: Matrix metalloproteinase-2 promotes $\alpha v \beta 3$ integrin-mediated adhesion and migration of human melanoma cells by cleaving fibronectin. PLoS One 7: e41591, 2012.

24. Chetty C, Vanamala SK, Gondi CS, Dinh DH, Gujrati M and Rao JS: MMP-9 induces CD44 cleavage and CD44 mediated cell migration in glioblastoma xenograft cells. Cell Signal 24: $549-559,2012$

25. Yu Q and Stamenkovic I: Cell surface-localized matrix metalloproteinase-9 proteolytically activates TGF-beta and promotes tumor invasion and angiogenesis. Genes Dev 14: 163-176, 2000.

26. Yu BF, Wu J, Zhang Y, Sung HW, Xie J and Li RK: Ultrasoundtargeted HSVtk and Timp3 gene delivery for synergistically enhanced antitumor effects in hepatoma. Cancer Gene Ther 20: 290-297, 2013. 
27. Hama R, Watanabe Y, Shinada K, Yamada Y, Ogata Y, Yoshida Y, Tamura T, Hiraishi T, Oikawa R, Sakurai J, et al: Characterization of DNA hypermethylation in two cases of peritoneal mesothelioma. Tumour Biol 33: 2031-2040, 2012.

28. Zhang H, Wang YS, Han G and Shi Y: TIMP-3 gene transfection suppresses invasive and metastatic capacity of human hepatocarcinoma cell line HCC-7721. Hepatobiliary Pancreat Dis Int 6: 487-491, 2007.

29. Tian H, Huang ML, Liu KY, Jia ZB, Sun L, Jiang SL, Liu W, McDonald Kinkaid HY, Wu J and Li RK: Inhibiting matrix metalloproteinase by cell-based timp-3 gene transfer effectively treats acute and chronic ischemic cardiomyopathy. Cell Transplant 21: 1039-1053, 2012.

30. Wu DW, Tsai LH, Chen PM, Lee MC, Wang L, Chen CY, Cheng YW and Lee H: Loss of TIMP-3 promotes tumor invasion via elevated IL- 6 production and predicts poor survival and relapse in HPV-infected non-small cell lung cancer. Am J Pathol 181: 1796-1806, 2012.

31. Ju HX, An B, Okamoto Y, Shinjo K, Kanemitsu Y, Komori K, Hirai T, Shimizu Y, Sano T, Sawaki A, et al: Distinct profiles of epigenetic evolution between colorectal cancers with and without metastasis. Am J Pathol 178: 1835-1846, 2011.

32. Lacy ER, Filippov I, Lewis WS, Otieno S, Xiao L, Weiss S, Hengst L and Kriwacki RW: p27 binds cyclin-CDK complexes through a sequential mechanism involving binding-induced protein folding. Nat Struct Mol Biol 11: 358-364, 2004.
33. Guo J, Ma Q, Zhou X, Fan P, Shan T and Miao D: Inactivation of p27kip1 promotes chemical hepatocarcinogenesis through enhancing inflammatory cytokine secretion and STAT3 signaling activation. J Cell Physiol 228: 1967-1976, 2013.

34. Ding WX, Ni HM, DiFrancesca D, Stolz DB and Yin XM: Bid-dependent generation of oxygen radicals promotes death receptor activation-induced apoptosis in murine hepatocytes. Hepatology 40: 403-413, 2004.

35. Hsu CH, Peng KL, Kang ML, Chen YR, Yang YC, Tsai CH, Chu CS, Jeng YM, Chen YT, Lin FM, et al: TET1 suppresses cancer invasion by activating the tissue inhibitors of metalloproteinases. Cell Rep 2: 568-579, 2012.

36. Wang J, Ding W, Sun B, Jing R, Huang H, Shi G and Wang H: Targeting of colorectal cancer growth, metastasis, and antiapoptosis in BALB/c nude mice via APRIL siRNA. Mol Cell Biochem 363: 1-10, 2012.

37. Bunz F, Dutriaux A, Lengauer C, Waldman T, Zhou S, Brown JP, Sedivy JM, Kinzler KW and Vogelstein B: Requirement for p53 and $\mathrm{p} 21$ to sustain $\mathrm{G}_{2}$ arrest after DNA damage. Science 282: 1497-1501, 1998

38. Smits VA, Klompmaker R, Arnaud L, Rijksen G, Nigg EA and Medema RH: Polo-like kinase-1 is a target of the DNA damage checkpoint. Nat Cell Biol 2: 672-676, 2000

39. Schwartz GK and Shah MA: Targeting the cell cycle: A new approach to cancer therapy. J Clin Oncol 23: 9408-9421, 2005. 